\title{
BRS AND ANTI-BRS INVARIANT STATES IN PATH INTEGRAL APPROACH TO HAMILTONIAN AND BIRKHOFFIAN MECHANICS
}

\author{
A.K.Aringazin \\ Department of Theoretical Physics \\ Karaganda State University \\ Karaganda 470074, Kazakhstan* \\ and \\ The Institute for Basic Research \\ P.O. Box 1577 \\ Palm Harbor, FL 34682, U.S.A.
}

Phys. Lett. B 314 (1993) 333-335

\begin{abstract}
Within the framework of recently proposed path integral approach to Hamiltonian and Birkhoffian mechanics, arising naturally to a fundamental supersymmetry lying behind the properties of the dynamical systems, we analyse BRS and anti-BRS invariant states. We found that among the all ghost sectors only $2 n$-ghost sector provides nontrivial physically relevant supersymmetric invariant solution, which was found earlier to have the Gibbs state form. We identify an algebra which is specific to Birkhoffian mechanics.
\end{abstract}

*Permanent address. 
Recently, Gozzi[1] proposed a path integral approach to Hamiltonian mechanics based on the formalism by Parisi and Sourlas [2][3] and Zinn-Justin[4] originally applied to stochastic systems. In a series of papers, Gozzi, Reuter, and Thacker [5]-[9] developed this application and found[5] that Hamiltonian mechanics indeed reveals a BRS-like supersymmetry lying behind the dynamical properties of the Hamiltonian systems. Conserved charges generating the supersymmetry form the algebra of inhomogeneous symplectic $I S p(2)$ group, and reflects symplectic geometry of phase space[5][6]. Associated supersymmetric invariant Hamilton function appeared to be a sum of the conventional Liouvillian and a term containing anticommuting (odd) variables so in the even part the formalism meets exactly the Liouvillian (operatorial) formulation of Hamiltonian mechanics constructed earlier by Koopman[10] and von Neumann[11]. States determined by probability density function, on which the Hamilton function acts, are defined on the extended phase space, which includes anticommuting variables - ghosts - in addition to the ordinary coordinates of the phase space $M_{2 n}$. Two conserved supersymmetric charges of a dynamical origin form, together with the Hamilton function, a supersymmetric algebra[6]. This supersymmetry has a close relation to the ergodicity[12]. Namely, it has been shown[6] that the BRS and anti-BRS invariant state, characterized by the ghost number $2 n$, is just a Gibbs state. So, when the supersymmetry is exact the system described by $2 n$-ghost density is in ergodic phase (chaos) while when the system is in regular motion phase the supersymmetry is always broken[6][7][13]. This gives a new criterion to detect transitions between chaotic and regular motion regimes in Hamiltonian systems[14].

In Hamiltonian mechanics, one deals with a constant fundamental symplectic 2 -form $\omega$. However, in general $\omega$ can be any closed and non-degenerate 2-form[15] [16]. The generalization of Hamiltonian mechanics based on generalized 2-form depending on coordinates of $M_{2 n}$ is reffered to as Birkhoffian mechanics [17]-[19]. Consistency of the Birkhoffian mechanics is provided by the Lie-isotopic construction [19][21][22]. It has been shown[23] that supersymmetry is still there, in the Birkhoffian generalization. In the $2 n$-ghost sector, it has been found[23] that the BRS and anti-BRS invariant state has the Gibbs state form. As it was mentioned[23] the solutions which are not in $2 n$-ghost sector might be as universal and important as the Gibbs state.

In this letter, we present results of the analysis[24] of all the non $2 n$-ghost sectors to find BRS and anti-BRS invariant solutions, both in Hamiltonian 
mechanics and the Birkhoffian generalization.

The associated Hamilton function has the form[5]

$$
\mathcal{H}=\omega^{i j} \partial_{j} H \partial_{i}+i \bar{c}_{i} \partial_{k}\left(\omega^{i k} \partial_{j} H\right) c^{j}
$$

Here, $H$ is Hamiltonian, $\omega^{i j}$ is a symplectic tensor, $a^{i}=\left(q^{i}, p_{i}\right)$ are phase space coordinates, $c^{k}$ and $\bar{c}_{i}$ are anticommuting variables, ghosts and antighosts respectively, $i, j, \ldots=1, \ldots, 2 n . \mathcal{H}$ is invariant under the supersymmetry generated by the following supersymmetric conserved charges[5]:

$$
Q=i c_{i} \partial_{i}, \quad \bar{Q}=i \bar{c}_{i} \omega^{i j} \partial_{j}, C=c^{i} \bar{c}_{i}, K=\frac{1}{2} \omega_{i j} c^{i} c^{j}, \bar{K}=\frac{1}{2} \omega^{i j} \bar{c}_{i} \bar{c}_{j}
$$

In addition to these charges, there are also two conserved nilpotent charges, for conserved Hamiltonian $H$,

$$
Q_{H}=e^{\beta H} Q e^{-\beta H}=Q-\beta N, \quad \bar{Q}_{H}=e^{-\beta H} \bar{Q} e^{\beta H}=\bar{Q}+\beta \bar{N} .
$$

where $N=c^{i} \partial_{i} H, \bar{N}=\bar{c}_{i} \omega^{i j} \partial_{j} H$, and $\beta$ is a real parameter. Their anticommutator closes on the Hamilton function[7][8], $\left[Q_{H}, \bar{Q}_{H}\right]=2 i \beta \mathcal{H}$. Supersymmetric invariant state $\rho(a, c)$ is the state annihilated by both $Q_{H}$ and $\bar{Q}_{H}$,

$$
Q_{H} \rho(a, c)=0, \quad \bar{Q}_{H} \rho(a, c)=0 .
$$

General representation of the density has the following form:

$$
\rho(a, c)=\sum_{k=0}^{2 n} \rho_{i_{1} \ldots i_{k}}(a) c^{i_{1}} \cdots c^{i_{k}} .
$$

Here, $\rho_{i_{1} \ldots i_{k}}(a)$ are totally antisymmetric functions. With the use of (3) and (5), the eqs. (4) take the form

$$
\begin{array}{r}
\sum_{k=0}^{2 n} c^{j} c^{i_{1}} \cdots c^{i_{k}} D_{j}^{-} \rho_{i_{1} \ldots i_{k}}(a)=0 \\
\sum_{p=0}^{2 n} \sum_{k=0}^{2 n} \alpha(p) c^{i_{1}} \cdots \delta_{j}^{i_{p}} \cdots c^{i_{k}} D_{+}^{j} \rho_{i_{1} \ldots i_{k}}(a)=0 .
\end{array}
$$

Here, we have denoted $D_{j}^{-}=\partial_{j}-\beta \partial_{j} H, D_{+}^{i}=\omega^{i j}\left(\partial_{j}+\beta \partial_{j} H\right)$, and $\alpha(p)=$ $+1(-1)$ for odd (even) $p$. Consistency of these two equations requires that 
to obtain non-trivial solutions one of the above equations should be satisfied identically.

(a) Even-ghost sector. There are only two possibilities to satisfy one of the eqs. (6)-(7) identically: (i) For $k=0$, the eq. (7) is satisfied identically so that the solution of the eq. (6) is $\rho(a, c)=\kappa \exp (+\beta H)$. This solution is evidently not physically reasonable $(\beta>0)$. (ii) For $k=2 n$, the eq. (6) is satisfied identically so that the solution of the eq. (7) is of the Gibbs form (cf. [6][7]), $\rho(a, c)=\kappa \exp (-\beta H) c^{1} \cdots c^{2 n}$. In the even-ghost sector, it appears to be useful to present the density $\rho(a, c)$ in the explicitly covariant form, $\rho=\rho_{m}(a) K^{m}, m=0, \ldots, n . K^{m}$ is invariant under Hamiltonian flow[15][23], $\mathcal{H} K^{m}=0$. With the aid of some algebra it is straightforward to calculate the commutators

$$
[K, \bar{N}]=N,[K, N]=0,\left[K^{m}, Q_{H}\right]=0,\left[K^{m}, \bar{Q}_{H}\right]=m K^{m-1}(Q+\beta N),
$$

so that the eqs. (4) take the form

$$
K^{m}(Q-\beta N) \rho_{m}(a)=0, \quad m K^{m-1}(Q+\beta N) \rho_{m}(a)=0 .
$$

The solution is of the Gibbs state form

$$
\rho(a, c)=\kappa e^{-\beta H} K^{n} .
$$

(b) Odd-ghost sector. There is no any possibility to satisfy identically one of the eqs. (6) and (7) in this case. Therefore, we are leaved only with trivial solution $\rho(a, c)=0$.

As the result, only $2 n$-ghost sector provides non-trivial physically relevant solution for BRS and anti-BRS invariant state which appears to be the Gibbs state (10).

To construct path integral approach to Birkhoffian mechanics one need to rerun the procedure[5] of evaluating the Hamilton function (1) but starting with the Birkhoff's equations[19], $\dot{a}^{i}(t)=\omega^{i j}(a) \partial_{j} H(a(t))$, instead of Hamiltonian ones. The dynamical supercharge $\bar{Q}_{H}$ has to be slightly modified $[23]$

$$
\bar{Q}_{H}=i \bar{c}_{i} \omega^{i j}(a) \partial_{j}-\frac{1}{2}\left(\partial_{k} \omega^{i j}(a)\right) c^{k} \bar{c}_{i} \bar{c}_{j}+\beta \bar{c}_{i} \omega^{i j}(a) \partial_{j} H .
$$

This charge can be casted into the following form:

$$
\bar{Q}_{H}=\bar{c}_{i} D_{+}^{i}-\frac{1}{2} f_{m}^{k l} c^{m} \bar{c}_{k} \bar{c}_{l},
$$


where $f^{i j k}=\omega^{i m} \partial_{m} \omega^{j k}$. In the field theoretical BRST technique, the operators placed similarly as $D_{+}^{i}$ in (12) play the role of the generators of some Lie group characterizing symmetry of the theory. It is easy to verify that the operators $D_{+}^{i}$ satisfy the commutation rule, $\left[D_{+}^{i}, D_{+}^{j}\right]=f^{i j k} D_{k}^{+}$, so that they constitute a Lie algebra. Note that this algebra is specific for Birkhoffian mechanics since $f^{i j k}=0$, in the Hamiltonian case.

It should be noted that there are cohomology classes of the solutions of the eqs. (4) that are characterized by different ghost numbers, due to nilpotency of the BRS and anti-BRS charges. The most interesting to identify are 0-, 1-, and $2 n$-ghost states: (i) Obviously, 0 - and $2 n$-ghost state are, respectively, anti-BRS and BRS invariant. (ii) For 1-ghost state, the condition $\bar{Q}_{H} \rho=0$ is equivalent to $D_{+}^{i} \rho=0$. This means that 1 -ghost state is antiBRS invariant if and only if it is $D_{+}^{i}$-invariant. However, it should be stressed that since it may occur that $\rho=\bar{Q}_{H} \chi$ ( $\chi$ has the ghost number two) there is no one-to-one correspondence between the space of $D_{+}^{i}$-invariant 1-ghost states and the space of cohomology class of 1-ghost states.

With the use of (11) and (5), the second eq. in (4) takes the form

$$
\begin{array}{r}
\sum_{p=0}^{2 n} \sum_{k=0}^{2 n} \alpha(p) c^{i_{1}} \cdots \delta_{j}^{i_{p}} \cdots c^{i_{k}} D_{+}^{j} \rho_{i_{1} \ldots i_{k}}(a) \\
-\frac{1}{2} c^{l} \sum_{p \neq q}^{2 n} \sum_{k=0}^{2 n} \alpha(p) \alpha(q) c^{i_{1}} \cdots \delta_{i}^{i_{p}} \cdots \delta_{j}^{i_{q}} \cdots c^{i_{k}} \omega^{i j}{ }_{,} \rho_{i_{1} \ldots i_{k}}(a)=0 .
\end{array}
$$

Again, we are leaved in effect to satisfy identically the first eq. in (4) because it implies itself the physically irrelevant solution. This can be done only with the choice $k=2 n$. It is a matter of tedious calculations to show that all the commutators (8) are still valid in the Birkhoffian case so that in the even ghost sector we have the only relevant solution, the Gibbs state (10). Furthermore, since $K^{n}$ is still invariant under Hamiltonian flow the solution (10) is defined only by the factor $\exp (-\beta H)$ so that it is indeed a Gibbs state (cf. [23]). 


\section{References}

[1] E.Gozzi, Phys. Lett. B 201 (1988) 525.

[2] G.Parisi and N.Sourlas, Phys. Rev. Lett. 43 (1979) 744.

[3] G.Parisi and N.Sourlas, Nucl. Phys. B 206 (1982) 321.

[4] J.Zinn-Justin, Nucl. Phys. B 275 (1986) 135.

[5] E.Gozzi, M.Reuter, W.D.Thacker, Phys. Rev. D 40 (1989) 3363.

[6] E.Gozzi, M.Reuter, Phys. Lett. B 233 (1989) 383.

[7] E.Gozzi, M.Reuter, Phys. Lett. B 238 (1990) 451.

[8] E.Gozzi, M.Reuter, Phys. Lett. B 240 (1990) 137.

[9] E.Gozzi, M.Reuter, in: Symmetries, Quarks and Strings, ed. M.Kaku et al. (World Sci., Singapore, 1991) p. 284.

[10] B.O.Koopman, Proc. Nat. Acad. Sci. USA 17 (1931) 315.

[11] J.von Neumann, Ann. Math. 33789 (1932) 587; 789.

[12] V.I.Arnold and A.Avez, Ergodic problems of classical mechanics (W.A.Benjamin Inc., New York, 1968).

[13] E.Gozzi, INFN preprint INFN/AE-91/20 (1991).

[14] E.Gozzi, M.Reuter, W.D.Thacker, INFN preprint INFN/AE-91/19 (1991).

[15] V.I.Arnold, Mathematical methods of classical mechanics (SpringerVerlag, New York, 1978).

[16] R.Abraham and J.Marsden, Foundations of mechanics (Benjamin, New York, 1978).

[17] G.D.Birkhoff, Dynamical systems (A.M.S.College Publ., Providence, 1927).

[18] R.M.Santilli, Hadronic J. 1 (1978) 223.

[19] R.M.Santilli, Foundations of theoretical mechanics, Vol.II: Birkhoffian generalization of Hamiltonian mechanics (Springer-Verlag, Heidelberg New York, 1983).

[20] R.M.Santilli, Hadronic J. Suppl. 4 A (1988) N 1. 
[21] A.K.Aringazin, A.Jannussis, D.F.Lopez, M.Nishioka, and B.Veljanoski, Algebras, Groups and Geometries 7 (1990) 211.

[22] A.K.Aringazin, A.Jannussis, D.F.Lopez, M.Nishioka, and B.Veljanoski, Santilli's Lie-isotopic generalization of Galilei's and Einstein's relativities (Kostarakis Publishers, Athens, 1991).

[23] E.Gozzi, M.Reuter, W.D.Thacker, INFN preprint INFN/AE-91/22 (1991).

[24] A.K.Aringazin, Hadronic J., submitted for publication. 